\title{
Considerations and Experience in the Treatment of Lung Cancer with VMAT SBRT + DIBH in Arms-Down Position
}

\author{
Yulin Song1*, Boris Mueller ${ }^{2}$, Kenneth Dow ${ }^{1}$, Ziad Saleh1, Xiaoli Tang1, Melissa Zinovoy², \\ Daphna Gelblum², Borys Mychalczak ${ }^{2}$
}

\author{
${ }^{1}$ Department of Medical Physics, Memorial Sloan Kettering Cancer Center, New York, USA \\ ${ }^{2}$ Department of Radiation Oncology, Memorial Sloan Kettering Cancer Center, New York, USA \\ Email: *songy@mskcc.org
}

\begin{abstract}
How to cite this paper: Song, Y.L., Mueller, B., Dow, K., Saleh, Z., Tang, X.L., Zinovoy, M., Gelblum, D. and Mychalczak, B. (2021) Considerations and Experience in the Treatment of Lung Cancer with VMAT SBRT + DIBH in Arms-Down Position. International Journal of Medical Physics, Clinical Engineering and Radiation Oncology, 10, 69-80.
\end{abstract}

https://doi.org/10.4236/ijmpcero.2021.1020 $\underline{07}$

Received: March 25, 2021

Accepted: May 21, 2021

Published: May 24, 2021

Copyright (๑) 2021 by author(s) and Scientific Research Publishing Inc. This work is licensed under the Creative Commons Attribution International License (CC BY 4.0).

http://creativecommons.org/licenses/by/4.0/

\begin{abstract}
The arms-up position is the most common treatment position adopted for lung cancer patients treated with radiation therapy. However, many elderly or frail patients have shoulder problems and cannot tolerate such an overstretched position for an extended period. Therefore, the arms-down position becomes the only alternative for this group of patients during radiation therapy. Even though the arms-down position is not ideal, it does provide a stable and comfortable patient immobilization position for radiation treatments that require a longer delivery time, such as stereotactic body radiation therapy (SBRT). In this study, we designed a protocol to treat lung cancer patients with VMAT stereotactic body radiation therapy (VMAT SBRT) and deep inspiration breath-hold (DIBH) in the arms-down position. Our initial clinical experience with this protocol indicates that it is reliable for patient immobilization and accurate in delivered dosimetry.
\end{abstract}

\section{Keywords}

Lung Cancer, VMAT, SBRT, DIBH, Arms-Down Position

\section{Introduction}

According to the latest U.S. national cancer statistics, there were approximately 235,760 new lung and bronchus cancer cases in 2021. Among them, $58.1 \%$ of the patients were diagnosed at the age of 75 and above, and $50.8 \%$ of the patients had distant metastases [1]. A significant proportion of these patients were therefore either medically inoperable or at high risk for thoracic surgery. For 
such patients, the primary treatment options are therefore limited to either chemotherapy, radiation therapy, or chemoradiation [2]. A subgroup of metastatic lung cancer patients may also benefit from targeted therapy depending on the mutation status of EGFR, ALK, ROS1, and BRAF [3] [4]. For medically inoperable patients with early-stage diseases, the results of several clinical studies have shown that stereotactic body radiation therapy (SBRT) and lobectomy achieve comparable primary tumor control rates and overall survival [5] [6] [7] [8]. SBRT is also indicated for elderly patients with poor pulmonary functions [9].

Currently, the most common treatment position for SBRT is the arms-up position, where the arms are above the head inside a thermoplastic mold. This treatment position offers several distinct advantages. First, it provides maximal spatial freedom for optimal beam placement, making treatment plans dosimetrically more conformal and steeper. Second, it completely spares the upper extremities by avoiding beams that enter and exit through the arms. Third, it makes body mold smaller and thereby easier to lock onto the treatment couch. Nevertheless, the arms-up position also has two intrinsic disadvantages. The first disadvantage is frequent involuntary arm and upper body movement, which is triggered by both arms being in an overstretched position. Such involuntary movements lead to target displacement and dosimetry perturbation. This phenomenon is further amplified by the combination of SBRT and deep inspiration breath-hold (DIBH). The combined modality paradigm prolongs the treatment time dramatically when delivered on machines without flattening filter-free (FFF) beams [10]. The use of volumetric modulated arc therapy (VMAT) has mitigated this negative impact on the treatment time. However, when many arcs, especially short arcs, are used in a treatment plan, the benefits of VMAT in delivery efficiency and low leakage dose become marginal and even diminish. The second disadvantage is that the arms-up position may preclude the use of long and full arcs for centrally and superiorly located targets. This is especially true for patients with a large physical build (requiring a broader thermoplastic mold) or for the treatment of a very posterior isocenter.

On the other hand, the arms-down treatment position is rarely used clinically. Nevertheless, it is the only reliable alternative for elderly and frail patients who are unable to tolerate the arms-up position for an extended period of time. However, the arms-down treatment position creates one significant challenge for SBRT plans delivered with rotational techniques such as VMAT and RapidArc ${ }^{\mathrm{TM}}$ (Varian Medical Systems, Palo Alto, CA, USA). It confines arcs to a much narrower space, thus degrading the target dose conformity and homogeneity. The issue becomes particularly pronounced when lesions invade the lateral side of the chest wall. The lateral lesions impede the use of lateral arcs because of their close proximity to the arms. From the perspective of setup reproducibility and treatment accuracy, the patient's comfort and stability on the treatment couch are crucial factors for realizing the precision of any SBRT immobilization devices and on-board imaging systems. 
In this study, a technique to treat lung cancer patients with VMAT SBRT + DIBH in the arms-down position was investigated. Our goals were to determine whether the arms-down position could produce treatment plans that are dosimetrically comparable to those for the arms-up position and still meet overall clinical objectives.

\section{Materials and Methods}

\subsection{Patient}

A 92-year-old patient was diagnosed with lung cancer of the right lower lobe. The patient was a former smoker with an 80-pack year history of tobacco use. The patient had the chronic obstructive pulmonary disease (COPD), atherosclerotic heart disease, cardiac arrhythmias, hyperlipidemia, and hypertension. A CT scan showed a $1.7 \mathrm{~cm}$ right lower lobe nodule. A PET scan showed low-grade uptake (SUV 2.0) in the nodule. The patient was not a good surgical candidate because of poor performance status and was therefore treated with definitive VMAT SBRT $(1200 \mathrm{cGy} \times 4)$ using a DIBH technique. Multiple DIBH sessions were performed during treatment.

\subsection{Patient Immobilization and CT Simulation}

The patient was placed in the supine position on a commercial lung SBRT board and immobilized with a tight custom Aquaplast mold. A rectangular hole was cut and marked in the abdominal area to place the Real-time Position Management ${ }^{\mathrm{TM}}$ gating block. The patient's arms were positioned to the sides with palms up and hands placed under the buttocks to keep them from moving during treatment. A four-dimensional CT (4DCT) scan was performed with a pulmonary $4 \mathrm{D}$ retrospective spiral protocol using a Brilliance Big Bore CT scanner (Philips Medical System, Cleveland, OH, USA). The scanner has an $85-\mathrm{cm}$ diameter bore, providing a maximum of $60-\mathrm{cm}$ field of view. Once a steady pulmonary waveform was observed, the scan was triggered.

The 4DCT scan was acquired from the thoracic inlet to the costophrenic angles. During the scan, the patient was instructed to breathe with a regular shallow pattern to reduce breathing artifacts in the reconstructed multi-phase images. Scan times ranged between 90 to $110 \mathrm{~s}$ for the 4DCT lung simulation. Once the $4 \mathrm{DCT}$ reconstruction was completed and maximum intensity projection (MIP) images were created, the internal target volume (ITV) was delineated slice-by-slice on the MIP images by the radiation oncologist using a volume drawing utility on the CT console. The ITV represented the trajectory of the moving gross tumor volume in space during a free-breathing cycle. The treatment isocenter was placed at the ITV center. Its coordinates were then sent to the positioning lasers for patient marking, as shown in Figure 1.

\subsection{Volume Delineation}

The treatment planning volumes were delineated on a DIBH CT scan in Eclipse 


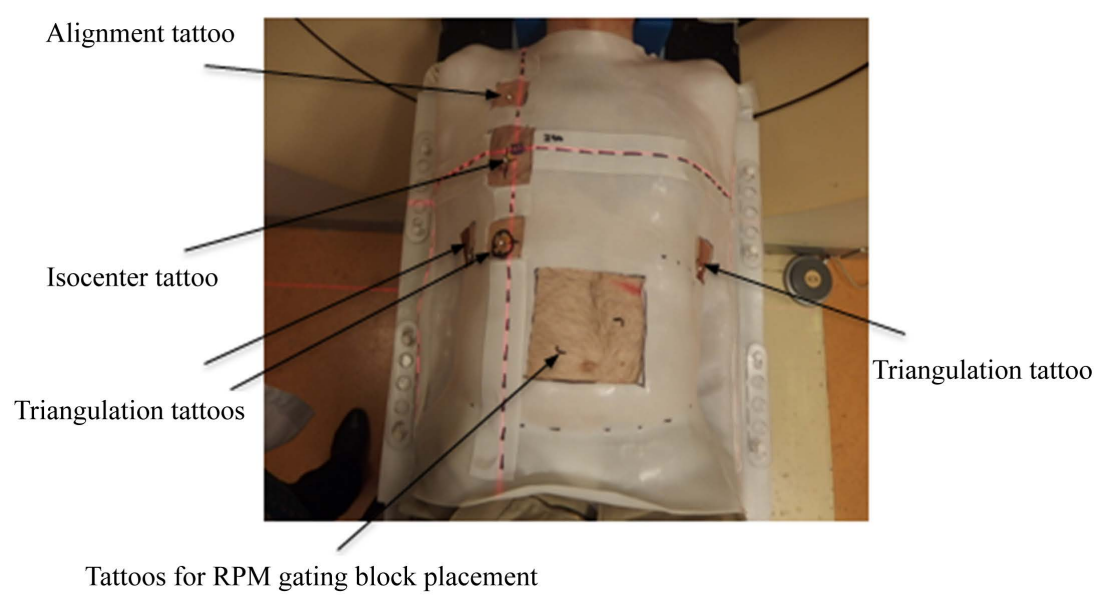

Figure 1. A custom body Aquaplast mold and patient marking tattoos.

(Varian Medical Systems, Palo Alto, CA). The planning target volume (PTV) was created by adding an appropriate setup uncertainty margin to the ITV. To minimize the electron build-up and build-down effects at the lung-tissue interfaces, an expanded PTV, referred to as "PTVE", was created by adding a uniform 3-mm margin to the PTV. The relevant organs at risk (OAR) were also delineated, including the carina, cord, esophagus, heart, liver, bilateral lungs, right arm, and right chest wall. To better spare them, a 3-mm uniform margin was added to the cord and esophagus. In addition, a series of tuning structures concentric to the PTV was created for dose tuning. The tuning structures had a $1-\mathrm{cm}$ increment in radius. By assigning proper dose constraints, these tuning structures could drive the higher isodose lines closer to the PTV, thus eliminating the undesirable hot spots in normal tissue and reducing the integral dose to the body as well (Figure 2). Furthermore, a large volume outside the PTV was created for normal tissue objective (NTO) calculation. The normal tissue objective is a unique feature of VMAT. It is a function of the distance from the PTV surface to the point of interest in the normal tissue. It is designed to limit the undesirable dose at that point in a physical manner.

\subsection{Beam Arrangement}

To maximally spare the right arm without compromising plan quality, the plan used six short partial arcs. Two arcs were placed anteriorly and four arcs were placed posteriorly. No arc entered the right arm. Only three arcs exited through a small portion of the medial side of the right arm for a short period of time. To enhance intensity modulation, control points were interleaved in gantry angles for each arc pair, i.e., each control point had a unique gantry angle. To improve patient comfort level during the DIBH treatment, all 6 arcs were short in span and low in Monitor Units (MU) so that each arc could be delivered in 2 to 3 DIBH sessions (Figure 3). During plan optimization, a proper dose constraint and priority level was applied to the right arm so that isodose lines would not be driven laterally by the optimizer. Table 1 lists the key beam parameters. 


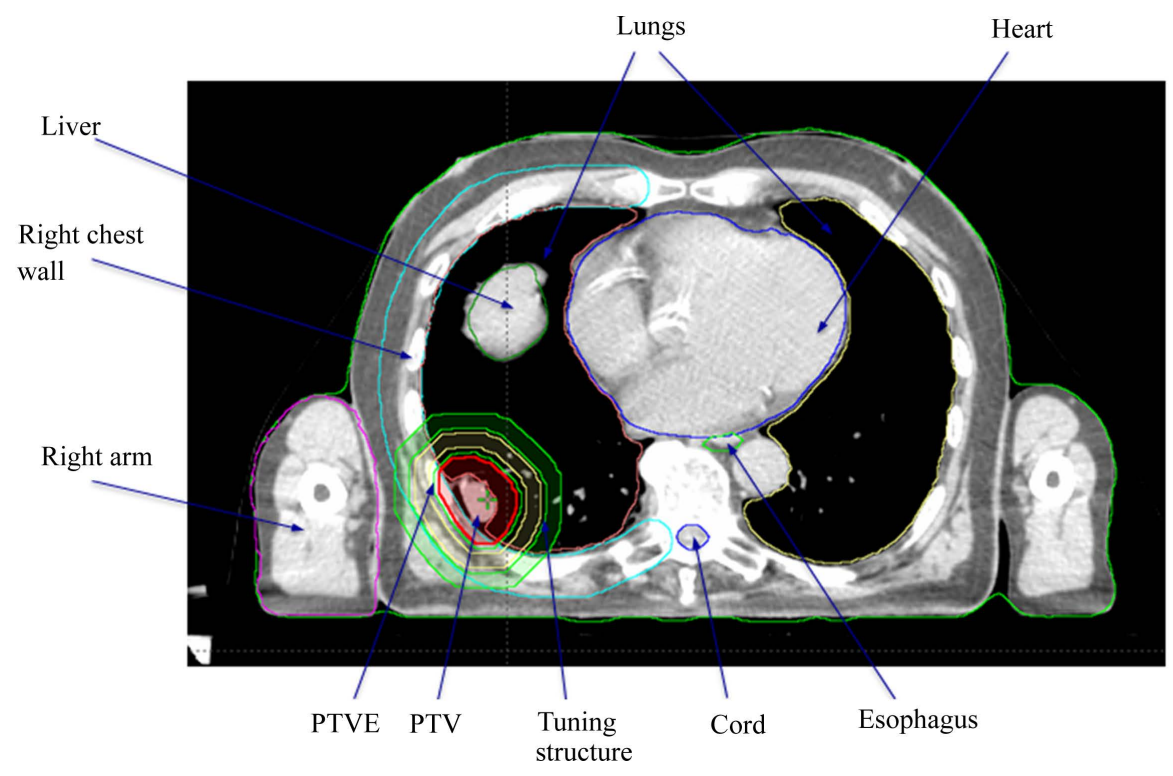

Figure 2. The planning target volume (PTV) and organs at risk were delineated on a deep inspiration breath-hold scan. In particular, the right arm was also delineated due to its close proximity to the PTV. PTVE = Expanded planning target volume.

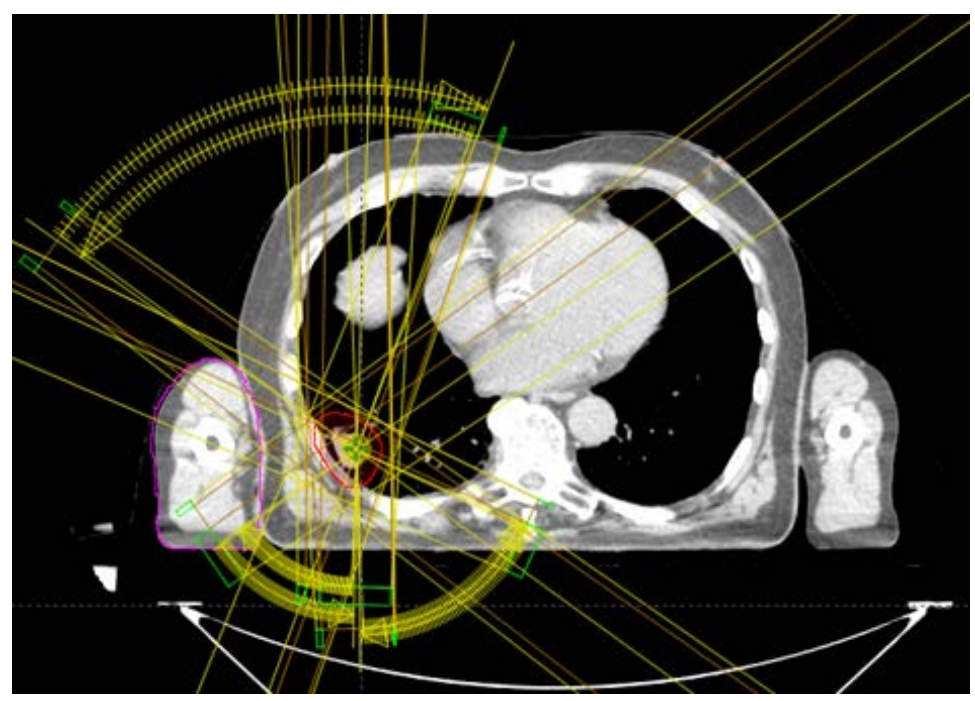

Figure 3. The VMAT plan consisted of 6 short arcs in an effort to maximally spare the right arm.

Table 1. Key beam parameters used in VMAT plan optimization.

\begin{tabular}{ccccc}
\hline Field No & Start Gantry Angle & End Gantry Angle & Rotation Direction & Field Weight \\
\hline Arc1 & $20^{\circ}$ & $305^{\circ}$ & CCW & 0.432 \\
Arc2 & $306^{\circ}$ & $21^{\circ}$ & CW & 0.435 \\
Arc3 & $115^{\circ}$ & $179^{\circ}$ & CW & 0.356 \\
Arc4 & $118^{\circ}$ & $114^{\circ}$ & CCW & 0.384 \\
Arc5 & $235^{\circ}$ & $181^{\circ}$ & CCW & 0.333 \\
Arc6 & $182^{\circ}$ & $236^{\circ}$ & CW & 0.336 \\
\hline
\end{tabular}




\subsection{VMAT SBRT Plan Delivery with DIBH}

Before treatment delivery, the patient was coached for DIBH. The patient was instructed to hold a deep breath steadily for about $20 \mathrm{~s}$ for each DIBH session so that each arc could be delivered within three DIBH sessions. After each DIBH session was completed, the patient was prompted to breathe freely for a short period of time and then continued with the next DIBH session. The DIBH gate window was set at $\pm 3 \mathrm{~mm}$.

\section{Results}

A representative arc control point aperture is shown in Figure 4. The red line represents the PTV, and the pink line indicates the right arm. The inner yellow outline defines the beam aperture for this particular control point. The outer yellow rectangle defines the four jaw positions. The $\mathrm{X} 1$ jaw blocks the beam from irradiating the right arm. The blue straight horizontal lines represent MLC. The unused 26th and 35th MLC leaf pairs were parked under the jaws. This technique significantly reduces the MLC interleaf end leakage. The digitally reconstructed radiography (DRR) was calculated with a soft tissue enhancement template, which was especially suitable for a soft tissue-based patient setup. For this particular arc, only a tiny medial portion of the right arm was irradiated for a short time period by the exiting beam during the gantry rotation.

Figure 5 shows the dose distribution in a representative axial plane, and Figure 6 shows the dose distribution in a representative coronal plane. As illustrated, the overall dose distribution was slightly stretched in the anteriorposterior direction. This was mainly attributed to the special arc arrangement and proper priority and constraint settings in optimization. This particular pattern of dose distribution created a steep dose gradient and rapid dose fall-off in the lateral direction, which maximally spared the right arm and the contralateral lung. These desirable dosimetric characteristics are exhibited in Figure 6. The $100 \%$ isodose line (yellow line) covered the PTV adequately. The $20 \%$ isodose

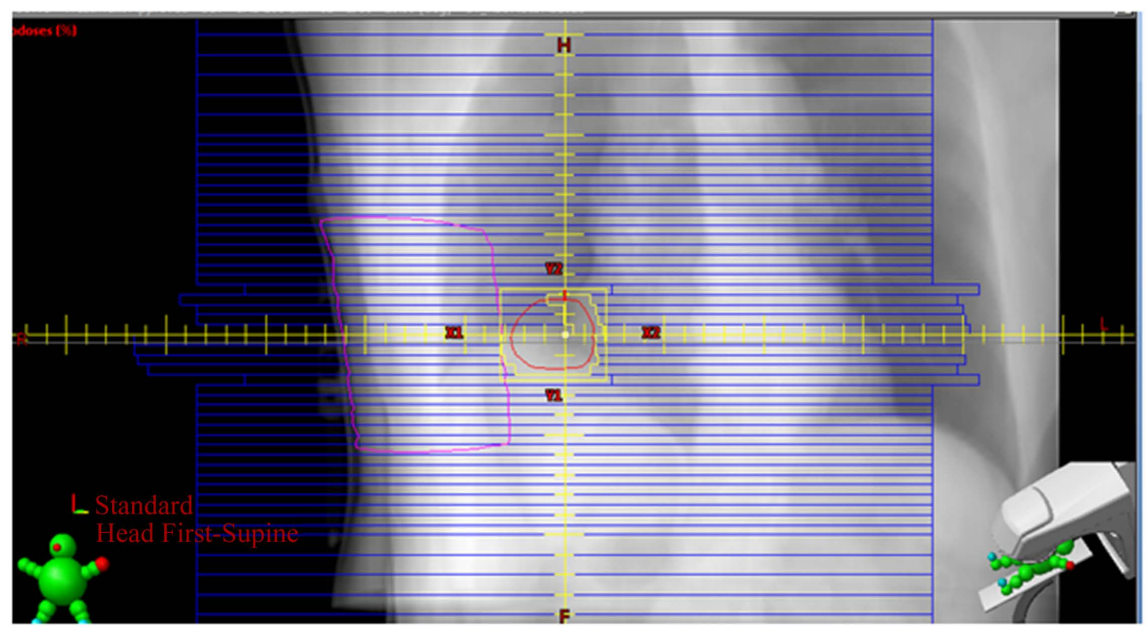

Figure 4. The aperture of a representative arc control point at a $0^{\circ}$ collimator angle. 


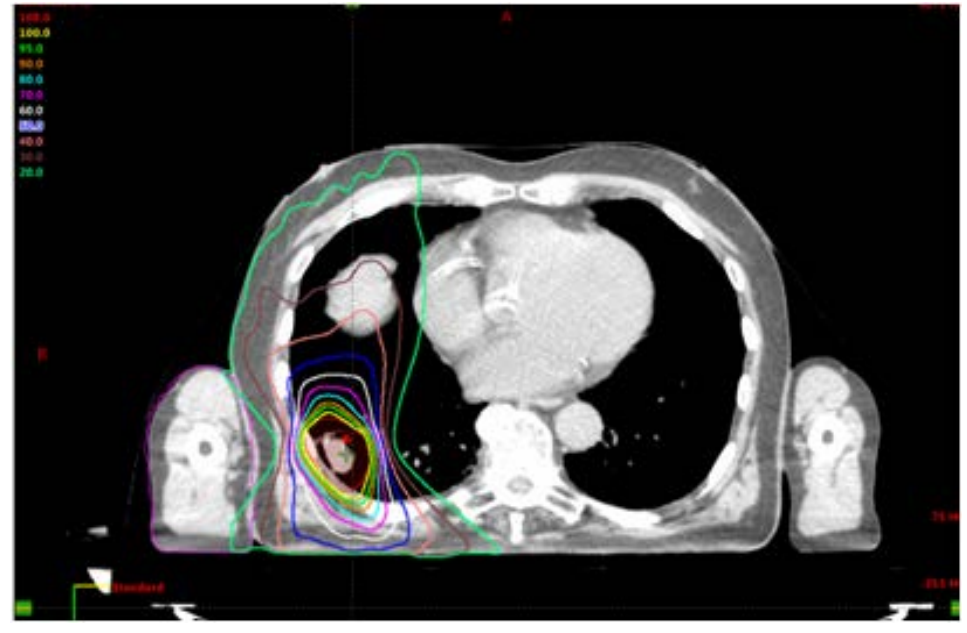

Figure 5. Dose distribution in a representative axial plane. The innermost red line represents the planning target volume, and the yellow line represents the $100 \%$ isodose line.

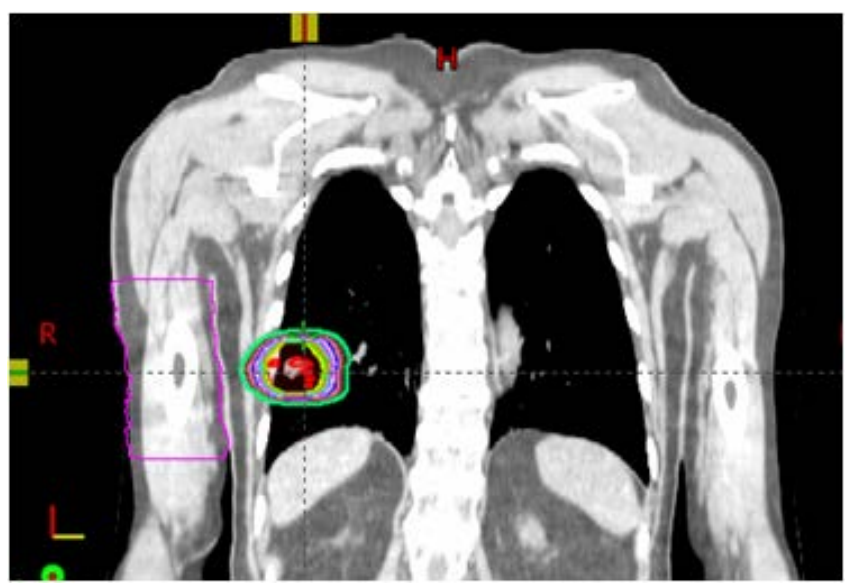

Figure 6. Dose distribution in a representative coronal plane. The lower isodose lines did not spread to the right arm and the contralateral lung.

line (green line) did not extend into a large volume of the right arm. The hot spot was limited to $108 \%$ and entirely confined to inside the PTV. The PTV dose conformity was 1.11 . One significant dosimetric advantage of this type of arc arrangement and dose distribution was that the delivered dosimetry was relatively immune to arm movement because the beams did not enter the right arm directly.

Figure 7 shows the treatment plan dose-volume histograms (DVH). All the dosimetric parameters met the institutional plan acceptance criteria. In particular, the total lung V20 was $2.86 \%$, significantly lower than the dose constraint of $12.5 \%$. The mean dose to the right arm was $93.2 \mathrm{cGy}$. It was observed that the arms-down plan was comparable to the arms-up plan in terms of PTV coverage, OAR sparing, and treatment time. The MUs for Arc1 to Arc6 were 527, 531, 434, 468,406 , and 409, respectively. The corresponding beam-on times were 53, 53, $43,47,43$, and $41 \mathrm{~s}$, which were completed in 2 or 3 DIBH sessions. 


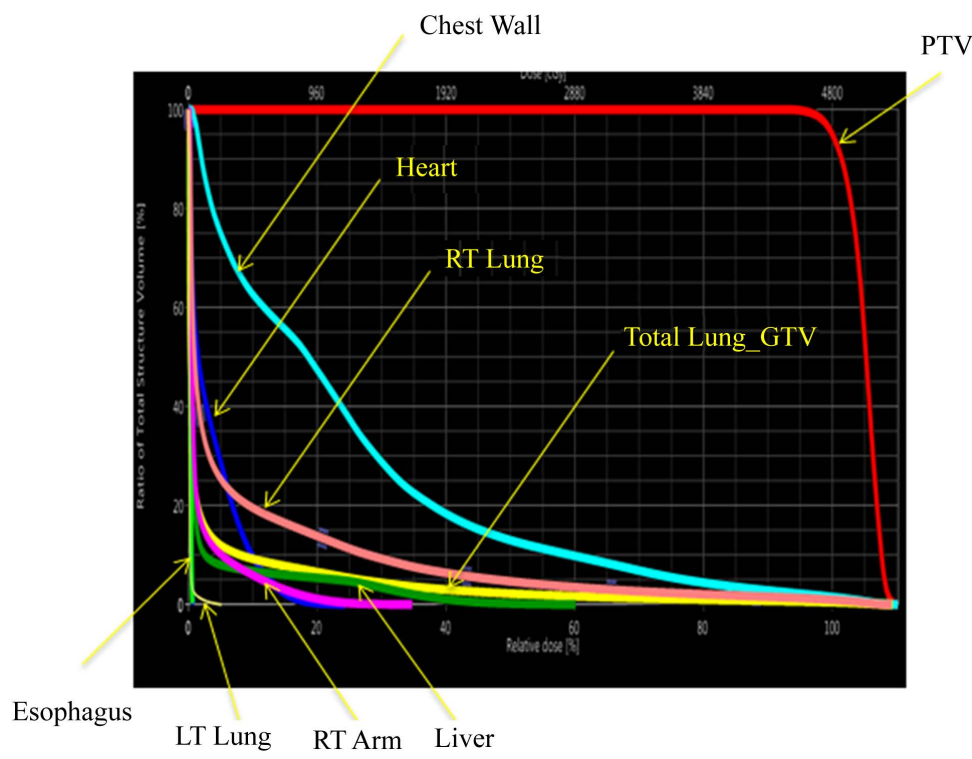

Figure 7. Plan dose-volume histograms. The $\mathrm{X}$-axis represents dose, and $\mathrm{Y}$-axis represents percentage volume. GTV = gross tumor volume.

\section{Discussion and Conclusions}

Under particular clinical circumstances, the arms-down position is the only safe and reliable way to treat lung cancer patients with VMAT SBRT. The central issue using this approach is to determine how to securely immobilize the arms so that Tissue-Maximum Ratio (TMR) perturbation can be minimized. Optimal arm positioning and good immobilization are the keys in the design of treatment plans whose beams enter through the arms. If the arms are not securely immobilized and move during the treatment, the TMR will change significantly, which will, in turn, impact the target dosimetry.

There are two strategies to minimize the probability of arm movement during radiation treatment. The first strategy is to improve patient comfort level after immobilization. This is especially important for elderly and frail patients. Patient arms are often overstretched or twisted by the immobilization device in an uncomfortable position to meet treatment planning requirements. This creates a scenario where the patient cannot relax and will fidget the arms and body during treatment. Our clinical experience is that the best method to immobilize the arms is to place the patient's hands under the buttocks with the palms up. This natural posture provides the highest comfort level for the patient. As a result, the patient can maintain this position consistently for a relatively long period. Compared with intensity-modulated radiation therapy (IMRT), VMAT is relatively insensitive to small variations in arm position. The smearing effect created by a long continuous arc can compensate for small perturbation in TMR.

The second strategy is to reduce the treatment time. This can be achieved in two ways. The first is to use a rotational treatment modality with aperture-based optimization. The second is to compute a treatment plan with less intensity modulation. Again, VMAT stands out among the competing treatment planning 
modalities in these two aspects. By adopting aperture-based optimization, VMAT plans can reduce the treatment time by up to $50 \%$ as a direct result of intensity modulation reduction [11]. If the target is far away from any OARs, then the MU minimization option can be used during plan optimization. This technique can further suppress intensity modulation and creates a plan with a total MU comparable to that computed by a dynamic conformal arc (DCA). Reducing the total number of arcs in a VMAT plan does not necessarily reduce the total MU for a given prescription. On the contrary, this approach may elevate the total MU due to hot spots.

Another effective technique to improve plan quality is to use interleaved control points in gantry angles, as illustrated in Table 1. If the first clockwise (CW) arc spans from $20^{\circ}$ to $305^{\circ}$, then the second counterclockwise (CCW) arc should span from $306^{\circ}$ to $21^{\circ}$ or $304^{\circ}$ to $19^{\circ}$. This methodology ensures that no control points in a pair of CW-CCW arcs share the same gantry angles and have identical beam apertures and $\mathrm{MU}$ weights. Interleaved control points significantly increase the range of intensity modulation and enhance the fineness of intensity modulation [12]. If the collimator angle of the CCW arc is also rotated by $90^{\circ}$, then the effect of intensity modulation could be further enhanced. Other collimator angles are generally not desirable for VMAT treatment planning because they will always lead to an increase in field sizes and result in a much higher MLC leakage dose.

With the arms-down position, the continuous long ipsilateral partial arcs that also cover the right arm are not optimal. Although this type of arc arrangement can improve target conformity and reduce the arc mode-up time on the Linac, it will inevitably deliver a lot of dose to the right arm. One practical option to resolve this dilemma is to insert an avoidance arc sector into the long arc at the arc span over the right arm. The avoidance arc sector can automatically pause the beam when the machine head is traversing the length of the arc over the right arm. However, the avoidance arc sector always occupies a certain number of valuable control points. The control points located in an avoidance arc sector are called the beamless control points, reflecting the fact that they do not deliver any dose to the target. To reduce the total plan MU and to make the plan delivery more time-efficient, the VMAT optimizer imposes a limit on the maximal number of control points allowed in an arc. For a $360^{\circ}$ full arc, the limit is 178 . Therefore, a longer arc yields a lower control point density, whereas a shorter arc always leads to a higher control point density. Because of the intrinsic interplay between the arc length and control point density, the total number of control points for a shorter arc is not substantially reduced as compared with a longer arc. This methodology automatically compensates for the beam angle sampling deficiency and guarantees an acceptable plan quality when shorter arcs are used in a plan. Our treatment planning philosophy for the arms-down position is to use multiple short partial arcs for targets located near the chest wall. This strategy could significantly elevate the control point density from 0.5 control points per degree for longer arcs to about 1.5 control points per degree for 
shorter arcs.

Nevertheless, due to the nature of rotational technique and, particularly, a much higher beam source sampling frequency than static modalities, the VMAT plan optimization time is relatively long compared to IMRT [13] [14] [15]. However, faster computers and more innovative algorithms will likely eliminate this deficiency over the next few years.

The radiation leakage caused by the MLC tongue-and-groove effect and rounded-leaf-end design is another drawback of the current version of the VMAT treatment planning system. In fact, this is a machine hardware constraint as opposed to a software limitation. This hardware deficiency can be significantly reduced by implementing a double-layer MLC design, with the upper MLC being placed right above the junction lines of the lower MLC.

Furthermore, even though VMAT is generally much faster than IMRT in plan delivery, the speed of plan delivery is still constrained by the gantry angular speed and machine dose rate. With the introduction of flattening filter-free beams, the dose rate is no longer the main bottleneck. Currently, the maximal gantry angular speed permitted by the various U.S. regulatory bodies is $6 \%$ due to patient safety concerns. Therefore, regardless of the dose rate used for plan delivery and total plan MU, the total treatment time for a VMAT SBRT plan is ultimately determined by the maximal gantry angular speed.

\section{Acknowledgements}

This research was funded in part by the NIH/NCI Cancer Center Support Grant P30 CA008748.

\section{Conflicts of Interest}

The authors declare no conflicts of interest regarding the publication of this paper.

\section{References}

[1] Surveillance, Epidemiology, and End Results Program (SEER), National Cancer Institute. (2021) Cancer Statistics Facts: Lung and Bronchus Cancer.

https://seer.cancer.gov/statfacts/html/lungb.html

[2] NCCN Clinical Practice Guidelines in Oncology. (2018) National Comprehensive Cancer Network (NCCN). Version 3, February 21, 2018. https://www.nccn.org

[3] Mok, T.S., Wu, Y.L., Ahn, M.J., Garassino, M.C., Kim, H.R., et al. (2017) Osimertinib or Platinum-Pemetrexed in EGFR T790M-Positive Lung Cancer. The New England Journal of Medicine, 376, 629-640. https://doi.org/10.1056/NEJMoa1612674 http://www.nejm.org/doi/full/10.1056/NEJMoa1612674

[4] Soria, J.C., Tan, D.S.W., Chiari, R., Wu, Y.L., Paz-Ares, L. et al. (2017) First-Line Ceritinib versus Platinum-Based Chemotherapy in Advanced ALK-Rearranged NonSmall-Cell Lung Cancer (ASCEND-4): A Randomized, Open-Label, Phase 3 Study. The Lancet, 389, 917-929. https://doi.org/10.1016/S0140-6736(17)30123-X https://www.sciencedirect.com/science/article/pii/S014067361730123X 
[5] Timmerman, R., Paulus, R., Galvin, J., Michalski, J., Straube, W., et al. (2010) Stereotactic Body Radiation therapy for Inoperable Early Stage Lung Cancer. JAMA, 303, 1070-1076. https://doi.org/10.1001/jama.2010.261 https://jamanetwork.com/journals/jama/fullarticle/185547

[6] Onishi, H., Shirato, H., Nagata, Y., Hiraoka, M., Fujino, M., et al. (2011) Stereotactic Body Radiotherapy (SBRT) for Operable Stage I Non-Small-Cell Lung Cancer: Can SBRT Be Comparable to Surgery? International Journal of Radiation Oncology, Biology, Physics, 81, 1352-1358. https://doi.org/10.1016/j.ijrobp.2009.07.1751 http://www.redjournal.org/article/S0360-3016(09)02787-4/fulltext

[7] Crabtree, T.D., Denlinger, C.E., Meyers, B.F., Naga, I. E., Zoole, J., et al. (2010) Stereotactic Body Radiation Therapy versus Surgical Resection for Stage I Non-Small Cell Lung Cancer. Journal of Thoracic and Cardiovascular Surgery, 140, 377-386. https://doi.org/10.1016/j.jtcvs.2009.12.054 http://www.jtcvsonline.org/article/S0022-5223(10)00261-8/abstract

[8] Chang, J.Y., Senan, S, Paul, M.A., Mehran, R., Louie, A.V., et al. (2015) Stereotactic Ablative Radiotherapy versus Lobectomy for Operable Sage I Non-Small-Cell Lung Cancer: A Pooled Analysis of Two Randomized Trials. The Lancet Oncology, 16, 630-637. https://doi.org/10.1016/S1470-2045(15)70168-3 https://www.sciencedirect.com/science/article/pii/S1470204515701683

[9] Grills, I.S., Mangona, V., Welsh, R., Chmielewski, G., McInerney, E. et al. (2009) Outcomes after Stereotactic Lung Radiotherapy or Wedge Resection for Stage I Non-Small Cell Lung Cancer. Journal of Clinical Oncology, 28, 928-935. https://doi.org/10.1200/JCO.2009.25.0928 http://ascopubs.org/doi/abs/10.1200/jco.2009.27.15_suppl.7516

[10] Scorsetti, M., Alongi, F., Castiglioni, S., Clivio, A., Fogliata, A., et al. (2011) Feasibility and Early Clinical Assessment of Flattening Filter Free (FFF) Based Stereotactic Body Radiotherapy (SBRT) Treatments. Radiation Oncology, 6, Article No. 113. https://doi.org/10.1186/1748-717X-6-113 https://ro-journal.biomedcentral.com/articles/10.1186/1748-717X-6-113

[11] Song, Y., Zhang, P., Obcemea, C., Mueller, B., Burman, C., and Mychalczak, B. (2009) The Development of a Novel Radiation Treatment Modality-Volumetric Modulated Arc Therapy. Conference Proceedings of the IEEE Engineering in Medicine and Biology Society, 2009, 3401-3404. https://www.ncbi.nlm.nih.gov/pubmed/19963576

[12] Song, Y., Obcemea, C., Mueller, B., Burman, C., and Mychalczak, B. (2012) A Systematic Approach to Patient Specific QA for Volumetric Modulated Arc Therapy (VMAT). Proceedings of World Congress on Medical Physics and Biomedical Engineering, 39, 1872-1875. https://doi.org/10.1007/978-3-642-29305-4_493 https://link.springer.com/chapter/10.1007/978-3-642-29305-4_493

[13] Song, Y., Zhang, P., Obcemea, C., Mueller, B., Burman, C., and Mychalczak, B. (2009) Dosimetric Effects of Gantry Angular Acceleration and Deceleration in Volumetric Modulated Radiation Therapy. Proceedings of World Congress on Medical Physics and Biomedical Engineering, 25, 1046-1050. https://doi.org/10.1007/978-3-642-03474-9_294 https://link.springer.com/chapter/10.1007/978-3-642-03474-9_294

[14] Song, Y., Mueller, B., Obcemea, C., and Mychalczak, B. (2011) Feasibility of Implementing Stereotactic Body Radiation Therapy Using a Non-Commercial Volumetric Modulated Arc Therapy Treatment Planning System for Early Stage Lung Cancer. 2011 Annual International Conference of the IEEE Engineering in Medicine and Biology Society, Boston, MA, USA, 30 August-3 September 2011, 409-412. 
https://doi.org/10.1109/IEMBS.2011.6090130

https://www.ncbi.nlm.nih.gov/pubmed/22254335

[15] Song, Y., Mueller, B., Obcemea, C., and Mychalczak, B. (2011) Image-Guided Volumetric Modulated Arc Therapy (IG-VMAT) for Prostate Cancer. Proceedings of the 5 th European Conference of the International Federation for Medical and Biological Engineering, 37, 223-226. https://doi.org/10.1007/978-3-642-23508-5_59

https://link.springer.com/chapter/10.1007/978-3-642-23508-5_59 